\title{
CRUSTÁCEOS DECAPODOS DA COSTA DO MARANHÃO, BRASIL
}

\author{
PETRONIO ALVES COELHO \\ Departamento de Oceanografia \\ Universidade Federal de Pernambuco, Recife, PE, Brasil \\ MARILENA RAMOS-PORTO \\ Departamemento de Pesca \\ Universidade Federal Rural de Pernambuco, Recife, PE, Brasil
}

\section{SYNOPSIS}

This paper presents a list of crustacean decapods occuring in the State of Maranharo coast, Brazil, based on the study of collections of the Departamento de Oceanografia (Universidade Federal de Pernambuco) and Laboratório de Hidrobiologia '(Fundacão Universidade do Maranhol) and on litterature records. There are recorded 149 species (37 species of Natantia and 112 species of Reptantia).

\section{Introdução}

A fauna de crustáceos do litoral do Maranhão apresenta especial importância por se encontrar na área de transição entre as províncias biogeográficas Brasileira e Guianense. Durante vários anos o Departamento de Oceanografia da Universidade Federal de Pernambuco (DO) reuniu material para o estudo desta fauna, consistindo principalmente em amostras recolhidas na plataforma continental pelo N/Oc. "Almirante Saldanha". Por outro lado, o Laboratório de Hidrobiologia da Fundação Universidade do Maranhão (LH) reuniu espécimens obtidos em coletas costeiras nos arredores de São Luis.

$\mathrm{O}$ estudo destas coleçð̄es mostrou que, apesar dos numerosos trabalhos anteriores, publicados desde 1894 até 1974 , a fauna de crustáceos estava mal conhecida e era bem mais rica do que se pensava.

A presente lista inclui todas as espécies estudadas pelos autores ou citadas na literatura. Para cada espécie são mencionados o nome científico válido, a ecologia, a localização do material estudado e as referências anteriores de sua ocorrência no Maranhão. São relacionadas, em anexo, espécies já encontradas tanto no $\mathrm{Pa}$ rá como no Piauŕ ou no Ceará, porém que não foram ainda coletadas no Maranhão.

Apesar de todo o esforço de coleta realizado até agora, ainda existem largos trechos do litoral do Maranhẫo por explorar e, por este motivo, novas coletas poderăo ampliar o conhecimento da fauna. A presente lista, no entantó, refere a ocorrência de 149 espécies de decápodos, sendo $37^{\prime}$ da subordem Natantia e 112 da subordem Reptantia. As famnlias com maior número de espécies são Penaeidae, Palaemonidae, Xanthidae e Majidae. Espécies de valor econômico atual ou potencial estão presentes nas familias Penaeidae, Palaemonidae, Palinuridae, Calappidae, Portunidae, Xanthidae, Grapsidae e Ocypodidae; a riqueza de espécies pertencentes a estas familias justificaria a realização de estudos, visando a avaliação dos estoques e a determinação das possibilidades de uma exploração econômica.

Foram encontrados problemas de identificação de algumas espécies, das quais quatro revelaram ser novas para a ciência. Fo publicada a descrição preliminar desta especies por um dos autores (Coelho) e as demais estão ainda em estudo.

Subordem NATANTIA

Família Penaeidae

Penaeus duorarum notiglis Perez Farfante. Areia, lama, 0-8- m; DO, LH. $(9,10,13,19)$.

Penaeus aztecus subtilis Perez Farfante. Areia, lama, 0-55 m; LH. $(12,13,19)$.

Penaeus schmitti Burkenroad. Areia, lama, 0-44 m; DO, LH. $(9,12,14,19)$.

Metapenaeopsis goodei (Smith). Rocha, cascalho, 0-60 m: DO. $(10)$.

Metapenaeopsis martinella Perez Farfante. Rocha, cascalho, $0-137 \mathrm{~m}$ : DO. $(9,14,15)$.

Trachypenaeus constrictus (Stimpson). 0-40 m; DO.

Xiphopenaeus kroyeri (Heller). Lama, areia, 0-70 m; LH. (19).

Sicyonia 'dorsalis Kingsley. Areia, lama, cascalho, 0-160 m; DO. $\left({ }^{9}\right)$.

Sicyonia parri (Burkenroad). Cascalho, 0-83 m; DO. $\left({ }^{9}\right)$.

Sicyonia laevigata Stimpson. Areia, cascalho, 0-85 m: DO, (9).

Solenocera geijskesi Holthuis. Lama, areia, 0-95 m; DO. $(9,10,17)$.

Solenocera atlantidis Burkenroad. Lama, 0-125 m.(17).
Mesopenaeus tropicalis (Bouvier). 30-915 m; (16). Hymenopenaeus sp. A. Areia, lama, 33-51 m; (9).

Familia Sergestidae

Acetes americanus Ortmann. Nerítico, epipelágico; DO.( $\left.{ }^{9}\right)$ Lucifer typus (Milne Edwards). Epipelágico. ${ }^{(1)}$

Lucifer faxoni Borradaile. Epipelágico ( ${ }^{1}$ ).

Familia Pasiphaeidae

Leptochela carinata Ortmann. Cascalho, 10-100 m; DO. ( $\left.{ }^{9}\right)$.

Leptochela serratorbita Bate. 0-67 m; DO. ( $\left.{ }^{y}\right)$.

Leptochela bermudensis Gurney. 11-59 m; DO.

Familia Disciadidae

Discias sp. A. Cascalho, $56-83 \mathrm{~m}$; DO. $\left({ }^{9}\right)$.

Família Palaemonidae

Brachycarpus sp. Cascalho, 31-131 m; DO. (9).

Macrobrachium amazonicum (Heller). Agua doce; DO, LH. (19).

Macrobrachium acanthurus (Wiegmann). Ảgua doce, estuários; DO, LH. $\left({ }^{19}\right)$.

Periclimenes americanus (Kingsley). Cascalho, 0-105 m; DO. ( 9 como $P$, americanus e $P$. sp. A).

Periclimenes longicaudatus (Stimpson). Cascalho, 0-100 m; DO. $(9$ como P. sp. B).

Pontonia margarita Smith. 0-44 m; DO. ( ${ }^{9}$ como Pontonia sp.).

Periclimenaeus ascidiarum Holthuis. Cascalho, areia, 0-73 m; DO. $\left({ }^{9}\right)$.

Periclimenaeus bermudensis (Armstrong). Cascalho, $49-75 \mathrm{~m}$; DO. $\left({ }^{9}\right)$.

Periclimenaeus sp. Cascalho. 33-72 m: DO.(9).

Anchistioides antiguensis (Schmitt). Cascalho, 9-83 m; DO. $\left({ }^{9}\right)$.

Família Alpheidae

Alpheus macrocheles (Hailstone). Cascalho, areia, lama, $53-90 \mathrm{~m}$; DO. $(9,18)$

Alpheus sp. LH. $\left({ }^{19}\right)$; obs.: espécimens não determináveis.

Alpheus heterochaelis (Say). Lama (ou manchas de lama entre rochas), águas rasas; LH. (19).

Alpheus cylindricus Kingsley. Cascalho, rocha, 0-80 m; DO. $\left({ }^{9}\right)$.

Familia Hippolytidae

Exhippolysmata oplophoroides (Holthuis). Areia, lama, $0-45 \mathrm{~m}$; DO.

Família Processidae

Processa spp. DO. $\left({ }^{9}\right)$; obs.: pelo menos cinco espécies, possivelmente novas para a ciência.

Subordem REPTANTIA

Familia Palinuridae

Panulirus argus (Latreille). Areia, cascalho, rocha, 0-64 m; DO. LH. (19). 
Panulirus laeyicauda (Latreille). Areia, cascalho, rocha, $0-46 \mathrm{~m} ;$ LH. (19).

Familia Callianassidae

Callichirus marginatus (Rathbun). Areia, lama, 46-290 m; DO. ( 9 como Callianassa).

Callichirus guara (Rodrigues). Lama, 0-43 m; DO. ( ${ }^{9}$ como Callianassa).

Familia Upogebiidae

Upogebia brasiliensis Holthuis. Estuários, rochas, águas rasas; DO. $(4,9,11)$.

Upogebia omissa Gomes Corrêa. Estuários, rochas, águas rasas; DO. $(4,9)$.

Família Paguridae Pylopagurus discoidalis (A. Milne Edwards). 95-229 m; DO.

Iridopagurus violaceus de Saint Laurent. Cascalho, 21-89 m; DO. $(4,9)$.

Familia Diogenidae

Paguristes erithrops Holthuis. Areia, cascalho, 0-53 m; DO $(4,9)$.

Dardanus venosus (Milne Edwards). Rocha, cascalho, areia. $0-100 \mathrm{~m}$; DO. $(2,4,9)$.

Petrochinus diogenes (Linnaeus). Areia, 0-72 m; DO. $(4,9)$.

Clibanarius vittatus (Bosc). Areia, lama, águas rasas; DO, LH.

Família Galatheidae

Munida simplex Benedict. Cascalho, 56-125 m, DO.

Munida brasiliae Coelho. Cascalho, $49-85 \mathrm{~m}$; DO.

Familia Porcellanidae

Pachycheles ackleianus A. Milne Edwards. Cascalho, 20-90 m; DO. $(4,9)$.

Petrolisthes armatus (Gibbes). Estuários, rocha, águas rasas. DO, LH. $(4,9,19)$.

Petrolisthes galathinus (Bosc). Estuários, rocha, 0-31 m; LH. (19).

Minyocerus angusaus (Dana). Comensal de Luida spp. (equinoderma), 0-59 m; DO. $(4,9)$.

Pisidia spp. DO. Possivelmente três espécies novas para a ciência.

Familia Albuneioae

Albunea paretiï Guerin. Areia, 0-90 m; $(4,9,12)$.

Familia Dromiidae

Hypoconcha sabulosa (Herbst). Are1a, cascalho, 0-39 m; DO. $(4,9)$.

Hypoconcha arcuata Stimpson. Areia, 0-82 m; DO. (24).

Familia Raninidae

Raninoides loevis (Latreille). Areia, lama, 16-67 m; DO. ( ${ }^{9}$ ). Symethis variolosa (Fabricius). Areia, cascalho, 19-110 m; DO. $(4,9)$.

Familia Dorippidae

Clythrocerus analogus Coelho, Cascalho, $56-110 \mathrm{~m}$; DO. $(4,7,9$ como $C . s p . A ; 6)$.

Ethusa mascarone americana A. Milne Edwards. Cascalho, areia, 20-69 m; DO. $(4,9)$.

\section{Famflia Palicidae}

Palicus affinis A. Milne Edwards \& Bouvier. Cascalho, 33-131 $\mathrm{m}$; DO. $(4,9)$.

Famnlia Calappidae

Calappa ocellata Holthuis. Areia, 0-46 m; DO, LH. (4, 9, 12,19 ).

Cycloes bairdii Stimpson. Cascalho, 13-120 m; DO. (4,9). Hepatus pudibundus (Herbst). Areia, lama, 0-49 m; LH. (19).

Osachila antillensis Rathbun. Cascalho, 80-140 m; DO. $(4,9)$.
Familia Portunidae

Portunus anceps (Saussure). Areia. 0-103 m; DO. $(4,9)$. Calinectes ornatus Ordway. Areia, lama, estuarios, 0-70 m; DO, LH. $(4,9,19,23)$

Callinectes bocourti A, Milne Edwards. Areia, lama, estuários, 0-20 m; L'H. $(19,23,26)$.

Callinectes exasperatus (Gerstaecker). Lama, areia, estuários, águas rasas, LH. (19).

Callinectes danae Smith. Estuários, águas rasas. (12).

Cronius ruber (Lamarck). Cascalho, areia, 17-105 m; DO $(4,9)$.

Familia Xantidae

Euryozius bouvieri (A. Milne Edwards). Cascalho, $51-120 \mathrm{~m}$ DO

Menippe nodifrons Stimpson. Rocha, estuários, águas rasas, DO, LH. $\left.{ }^{19}\right)$.

Coralliope spinipes (A. Milne Edwards). Cascalho, rocha, 0-82 m; DO.

Micropanope pusilla A. Milne Edwards. Areia, cascalho, 21-70 m; DO.

Micropanope nuttingi (Rathbun). Cascalho, 0-118 m; DO

Micropanope urinator (A. Milne Edwards). Cascalho,

$75-85 \mathrm{~m} ; \mathrm{DO}$.

Paractaea rufopunctata nodosa (Stimpson). Cascalho, areia, 15-120 m; DO.

Actaea acantha (Milne Edwards). Cascalho, 54-125 m; DO.

Banareia palmert (Rathbun). Cascalho, 47-125 m; DU

Lophopanopeus sp. Cascalho, 8-103 m; DO.

Panopeus herbstii Milne Edwards. Rocha, estuários, águas rasas, LH.(19).

Panopeus americanus Saussure. Rocha, estuários, águas rasas, LH. (19).

Panopeus occidentalis Saussure. Rocha, estuários, águas rasas, LH. (19).

Panopeus hartï Smith. Rocha, estuários, 0-24 m; DO.

Hexapanopeus paulensis Rathbun. Areia, lama, rocha, estuários, 0-47 m; DO. LH. (19).

Eurytium limosum (Say). Areia, rocha, estuários, lama, águas rasas, DO, LH. $(19,25)$

Xanthias inornatus (Rathbun). Cascalho, 61-75 m; DO.

Pilumnus caribaeus Desbonne \& Schramm. Areia, lama, rocha, 0-53 m; DO.

Pilumnus reticulatus Stimpson. Areia, cascalho, 23-75 m; DO.

Pilumnus diomedeae Rathbun. Cascalho, 76-120 m; DO.

Melybia thalamita Stimpson. Cascalho, 31-118 m; DO.

Família Goneplacidae

Euryplax nitida Stimpson. Areia, cascalho, 0-47 m; DO

Família Majidae

Euprognatha gracilipes. A. Milne Edwards. Cascalho, 69-305 m; DO.

Batrachonotus brasiliensis Rathbun. Areia, cascalho, $12-73 \mathrm{~m}$; DO. $(4,5,9)$.

Collodes armatus Rathbun. Cascalho, 75-92 m; DO

Collodes inermis. A. Milne Edwards. Areia, lama, 25-105 m; DO $(4,5,9,12)$.

Podochela algicola (Stebbing). Cascalho, 24-110 -m; DO. $(4,5,9)$.

Stenorhynchus seticornis (Herbst). Cascalho, rocha, areia, $0-93 \mathrm{~m}$; DO. $(4,5,9,20,22)$.

Inachoides forceps A. Milne Edwards. Areia, lama, 0-68 m; DO. $(4,9)$.

Pitho lherminieri (Schramm). Cascalho, areia, 9-49 m: DO. $(4,5,9)$.

Picroceroides tubularis Miers. Cascalho, 39-90 m; DO. (4, 5, 9). Epialtoides rostratus Coelho. Cascalho, $19-54 \mathrm{~m}$; DO. Mocosoa crebripunctata Stimpson. Cascalho, 37-131 m: DO. $\left({ }^{9}\right)$.

Notolopas brasiliensis Miers. Areia, lama, 0-82 m: DO. $(4,5,9)$.

Pelia rotunda A. Milne Edwards. Areia, cascalho, 0-82 m; DO $4,5,9,22$ )

Libinia ferreirae Brito Capello. Lama, 11-27 m (12).

Hemus cristülipes A. Milne Edwards. Cascalho, 26-110 m; DO. $(4,5,9)$.

Mithrax (Mithrax) cornutus Saussure. Cascalho, 53-75 m; DO. 
Mithrgx (Mithrax) acuticornis Stimpson. Cascalho, 35-120 m;
DO. $(4,5,9)$. DO, $(4,5,9)$.

Mithrax (Mithrax) hispidus (Herbst). Rocha, cascalho, 0-69

Mithrax (Mithraculus) forceps (A. Milne Edwards). Rocha,

Microphrys bicormutus (Latreille). Areia, rocha, águas rasas. Macrogoeloma eutheca (Stimpson). Cascalho, 30-131 m; DO.
$(4,5,9)$.

Macrogoeloma concavum Miers. Cascalho, $13-80 \mathrm{~m}$; DO. Macrocoeloma sp. Cascalho, 44-83 m; DO.

Leptopisg setirostris (Stimpson). Cascalho, areia, 22-75 m; DO. $(4,5,9)$.

Familia Parthenopidae

Parthenope (Platylambrus) fratercula (Stimpson). Areia, cascalho, 77-118 m: DO.

Parthenope (Platylambrus) serrata (Milne Edwards). Areia, lama, $35-75 \mathrm{~m}$; DO.

Thyrolambrus astroides Rathbun. Cascalho, 46-85 m; DO

Cryptopodia concava Stimpson. Cascalho, areia, $17-73 \mathrm{~m}$ DO.

Heterocrypta lapidea Rathbun. Areia, cascalho, 16-49 m; DO.

Família Leucosiidae

Ebalia stimpsonii. A. Milne Edwards. Areia, cascalho, 13-83 m; DO. $(4,9)$

Ebalia vertiginosa Coelho. Cascalho, 23-92 m; DO. (6).

Speloeophorus elevatus Rathbun. Cascalho, 21-83 m; DO. $\left({ }^{4}, 9\right)$.

Persephona finneganae Rathbun. Lama, 12-70 m; DQ. 4 4, 9). Callidactylus asper Stimpson. Cascalho, 27-81, DO. (4,9).

Mliacanthe sparsa Stimpson. Cascalho, 23-90 m; DO. $(4,9)$.

Iliacantha intermedia Miers. Areia, cascalho, 20-35 m; DO $(4,9)$.

Iliacantha subglobosa Stimpson. Areia, cascalho, 72-109 m; DO.

Familia Grapsidae

Goniopsis cruentata cruentata (Latreille). Estuários, águas; LH. (19).

Pachygrapsus transversus (Gibbes). Rocha, estuários, águas rasas; LH. (19).

Pachygrapsus gracilis (Saussure). Rocha, estuários, águas rasas; DO.

Euchirograpsus sp. Cascalho, $75 \mathrm{~m}$; DO.(9).

Aratus pisonii (Milne Edwards). Estuários, águas rasas; LH (19)

Sesarma (Holometopus) rectum Randall. Estuários, águas rasas; DO. LH. (19).

Familia Pinnotheridae

Pinnotheres sp. Perto de maculatus). Areia. $32 \mathrm{~m}$; DO. $(4, y)$. Pinnixa sp. B. Areia, $21 \mathrm{~m}$; DO. $(4,9)$.

Parapinnixa hendersoni Rathbun. Areia, 38-46 m; DO. ( ${ }^{9}$ ).

Familia Ocypodidae

Ocypode quadrata (Fabricius). Areia; DO, LH. $(4,9,19)$.

Uca maracoani (Latreille). Estuários, águas rasas; DO, LH.

Uca mordax (Smith). Estuários, águas rasas; DO.

Uca thayeri Rathbun. Estuários, águas rasas; LH. (19) Uca leptodactyla Rathbun. Estuários, águas rasas; DO, LH.

Ucides cordatus(Linnaeus). Estuários; LH. $(19,25)$.

Família Hapalocarcinidae

Troglocarcinus corallicola Verrill. Comensal de corais, 0-75 $\mathrm{m} ; \mathrm{DO} .\left({ }^{4,9}\right)$.

\section{ANEXO}

Espécies encontradas no Pará e no Piauí, ou Ceará, porém ainda não assinaladas para o Maranhão.
Trachycaris restricta (A. Milne Edwards)

Scyllarus chacei Holthuis

Scyllarides delfosi Holthuis

Porcellana sayana (Leach)

Megalobrachium mortenseni Haig

Clythrocerus carinatus Coelho

Pilumnus quoyi Milne Edwards

Pannoplax depressa Stimpson

Aepinus septemspinosus (A. Milne Edwards)

Podochela gracilipes Stimpson

Parthenope (Parthenope agona (Stimpson)

Parthenope (Plathylambrus) pourtalesii (Stimpson)

Mesorhoea sexspinosa (Stimpson)

Ebalia conica Coelho

Ebalia obliqua Coelho

Persephona punctata (Linnaeus)

\section{Bibliografia}

1. BARTH, R. \& COSTA, P. F. 1968. Estudos sobre os Leuciferinae na região entre Recife e Cabo Orange, Publçōes Inst. Pesq. Mar., R. de J., (30): 1-25.

2. BIFFAR, T. A. \& PROVENZANO Jr., A. J. 1972 Biological results of the University of Miami Deep-Sea Expedition. 94. A reexamination of Dardanus venosus (H. Milne Edwards) and $D$. Imperator (Miers), with a description of a new species of Dardamus from the Western Atlantic (Crustacea, Decapoda, Diogenidae). Bull, mar. Sci, 22(4): 777-805.

3. BULLIS, H. R. \& THOMPSON, J. R. 1965. Collections by the exploratory fishing vessels Oregon, Silver Bay, Combat and Pelican made during 1956-1960 in the south western North Atlantic Spec. scient. Rep. Fish. U. S. Fish Wildl. Serv., (510): $1-130$

4. COELHO, P. A. 1971. A distribuição dos crustáceos decápodos reptantes do Norte do Brasil, Trabhs Inst. oceanogr., Univ. Fed. Pernambuco, 9/11: 223-238.

5. - - - 1971. Nota prévia sobre os Majidae do Norte Nordeste do Brasil. (Crustacea, Decapoda); Arq. Mus. nac. Rio de J., 54:137-143.

6. --_-_- 1973. Descrição preliminar de Ebalia vertiginosa, n. sp., do Norte e Nordeste do Brasil (Crustacea, Decapoda, Leucosiidae), Ciênc. Cult., S. Paulo, 25 (6): 343.

7. -- 1973. Descrição preliminar de Clythrocerus analogus, n. sp., do litoral brasileiro (Crustacea, Decapoda, Dorippidae). Ciênc. Cult., S. Paulo, 25 (6):343-344.

8. --- - 1973. Descrição de três espécies novas de Majidae do Brasil (Decapoda, Brachyura). Trabhs oceanogr., Univ. Fed. Pernambuco, 13:119-132

9. - - - \& RAMOS, M. A. 1973. A constituição e a distribuição da fauna de decápodos do litoral leste da América do Su entre as latitudes de $50 \mathrm{~N}$ e $390 \mathrm{~S}$. Trabahs oceanogr., Univ. Fed. Pernambuco, 13:133-236.

10. - - - \& - - - 1973. Contribuição ao conhecimento dos camarões comerciais do Norte e Nordeste do Brasil. XXXVIII Comissão Oceanográfica N/Oc. "Almirante Saldanha" (5/11 a 20/12/68. D. H. N., D. G., 26-XIV,: 123-129.

11. GOMES CORREA, M. M. 1968 . Sobre as espécies de "Upogebia" Leach do litoral brasileiro. com descriçâo de uma espécie nova. Revta bras. Biol, 28(2): 97-10̄0.

12. NOMURA, H. \& FAUSTO FILHO, J. 1966. Shrimp survey in coastal and offshore waters of Northeastern and Northern Brazil. Arq. Estç. Biol. mar., Univ. Fed. Ceará, 6 (1): 15-29.

13. PEREZ FARFANTE, I. 1967. A new species and two subspecies of shrimp of the genus Penaeus from the western Atlantic. Proc. biol. Soc. Wash., 80: 83-99.

14. - - 1969. Western Atlantic shrimps of the genus Penae sus. Fishery Bull. Fish Wildl. Serv. U. S., 67(3):461-591.

15. - - - 1971. Western Atlantic shrimps of the genus Metape naeopsis, with descriptions of three new species (Crustacea: Decapoda: Penaeidae). Smithson. Contr. Zool., 79:1-37.

16. - 1977. American solenocerid shrimps of the genera Hymenopenaeus, Haliporoides, Pleoticus, Hadropenaeus new genus, and Mesopenaeus, new genus. Fishery Bull. Natn mar. Fish. Serv., 75(2): 261-346.

17. - - - \& BULLIS, Jr., H. R. 1973. Western Atlantic shrimps of the genus Solenocera with description of a new species (Crustacea: Decapoda: Penaeidae). Smithson. Contr. Zool. 153:1-33.

18. RAMOS-PORTÓ, M. 1979. Ocorrência de Alpheus macrocheles (Hailstone) (Crustacea: Decapoda: Alpheidae) no Norte e Nordeste do Brasil. Trabhs oceanogr., Univ. Fed. Pernambuco, $14: 117-130$. 
19. - - - ;ERREIRA-CORREIA, M. M. \& SOUZA, N. R. Crustáceos da Illha de São Luís (Estado de Maranhão, Brasil). Bolm Lab. hidrobiol.. Fund. Univ. Maranhão. (no prelo). 20. RATHBUN, M. J. 1984. Note on the crabs of the tamily Inachidae in the United Stakes Museum. Proc. U. S. ntn. Mus., 17: 43-75.

21. - The grapsoid crabs of America. Bull. U. S. natn. Mus., 97: 1-461.

22. - - 1925. The spider crabs of America. Bull. U. S. natn. Mus., 129:1-613.
23. - - - - 1930. The cancroid crabs of America of the families Euryalidae, Portunidae, Atelecyclidae, Cancridae and Xanthidae. Bull. U. S. natn. Mus., 152:1-609.

24. RATHBUN, M. J. 1937. The oxystomatous and allied crabs of America. Bull. U. S. natn. Mus., 166:1-277.

25. TURKAY, M. 1970. Die Gecarcinidae Amerikas. Mit einem Anhang über Ucides Rathbun. Senckenberg. biol, 51(5/6): 333-354.

26. WILLIAMS, A. B. 1974 . The swimming crabs of the genus Callinectes (Decapoda: Portunidae). Fishery Bull. Natn mar. Fish. Serv., 72(3): 685-798. 